\title{
Quadratic forms of multivariate skew normal-symmetric distributions is
}

\author{
Wen-Jang Huang*, Yan-Hau Chen \\ Department of Applied Mathematics, National University of Kaohsiung, Kaohsiung 811, Taiwan
}

Received 29 June 2005; received in revised form 30 September 2005

Available online 15 November 2005

\begin{abstract}
Following the paper by Gupta and Chang (Multivariate skew-symmetric distributions. Appl. Math. Lett. 16, 643-646 2003.) we generate a multivariate skew normal-symmetric distribution with probability density function of the form $f_{\boldsymbol{Z}}(\boldsymbol{z})=2 \phi_{p}(z ; \boldsymbol{\Omega}) G\left(\boldsymbol{\alpha}^{\prime} \boldsymbol{z}\right)$, where $\boldsymbol{\Omega}>0, \boldsymbol{\alpha} \in \mathbb{R}^{p}, \phi_{p}(z ; \boldsymbol{\Omega})$ is the $p$-dimensional normal p.d.f. with zero mean vector and correlation matrix $\boldsymbol{\Omega}$, and $G$ is taken to be an absolutely continuous function such that $G^{\prime}$ is symmetric about 0 . First we obtain the moment generating function of certain quadratic forms. It is interesting to find that the distributions of some quadratic forms are independent of $G$. Then the joint moment generating functions of a linear compound and a quadratic form, and two quadratic forms, and conditions for their independence are given. Finally we take $G$ to be one of normal, Laplace, logistic or uniform distribution, and determine the distribution of a special quadratic form for each case.

(C) 2005 Elsevier B.V. All rights reserved.
\end{abstract}

MSC: primary 62H10; secondary 62E15

Keywords: Chi-square distribution; Independence; Moment generating function; Non-normal models; Quadratic form; Skew normal distribution; Skew symmetric distribution

\section{Introduction}

The univariate skew normal distribution was introduced by Azzalini (1985, 1986), and Gupta et al. (2004b), and its multivariate version by Azzalini and Dalla Valle (1996), Azzalini and Capitanio (1999), Gupta and Kollo (2000), and Gupta et al. (2004a). These classes of distributions include the normal distribution and have some properties like the normal and yet is skew. They are useful in studying robustness. Here a $p$-dimensional random vector $\boldsymbol{Z}$ is said to have a multivariate skew normal distribution if it is continuous and its probability density function (p.d.f.) is given by

$$
f_{\boldsymbol{Z}}(\boldsymbol{z})=2 \phi_{p}(\boldsymbol{z} ; \boldsymbol{\Omega}) \Phi\left(\boldsymbol{\alpha}^{\prime} \boldsymbol{z}\right)
$$

\footnotetext{
Support for this research was provided in part by the National Science Council of the Republic of China, Grant No. NSC 93-2118-M390-001.

*Corresponding author. Tel.: + 88675919169 ; fax: 88675919360.

E-mail addresses: huangwj@nuk.edu.tw (W.-J. Huang), m0934416@mail.nuk.edu.tw (Y.-H. Chen).
} 
where $\boldsymbol{\Omega}>0, \boldsymbol{\alpha} \in \mathbb{R}^{p}, \phi_{p}(\boldsymbol{z} ; \boldsymbol{\Omega})$ is the p.d.f. of $N_{p}(\mathbf{0}, \boldsymbol{\Omega})$ distribution (the $p$-dimensional normal distribution with zero mean vector and correlation matrix $\boldsymbol{\Omega})$, and $\Phi(\cdot)$ is the standard normal cumulative distribution function (c.d.f.). It is denoted by $\boldsymbol{Z} \sim S N_{p}(\boldsymbol{\Omega}, \boldsymbol{\alpha})$, to mean that the random vector $\boldsymbol{Z}$ has $p$-variate skew normal p.d.f. (1). Quadratic forms of skew normal random vectors have been studied by many authors, including Azzalini (1985), Azzalini and Dalla Valle (1996), Azzalini and Capitanio (1999), Loperfido (2001), Genton et al. (2001), and Gupta and Huang (2002).

The classes of univariate symmetric p.d.f.s which depend on a skewness parameter have also been studied by Gupta et al. (2002), and Arellano-Valle et al. (2004). In particular the skew normal, uniform, Student's $t$, Cauchy, Laplace, and logistic distributions are given and some of their properties are explored. The multivariate skew-Cauchy distribution and multivariate skew $t$-distribution are studied by Arnold and Beaver (2000), and Gupta (2003), respectively. Following Gupta et al. (2002), Nadarajah and Kotz (2003) studied univariate skewed distributions generated by the normal kernel. More precisely, they generated skew p.d.f.s of the form $2 f(u) G(\lambda u)$, where $f$ is taken to be a normal p.d.f. with zero mean, while the c.d.f. $G$ is taken to come from one of normal, Student's $t$, Cauchy, Laplace, logistic or uniform distribution. A class of multivariate skew distributions has been introduced by Gupta and Chang (2003).

Following Gupta and Chang (2003), the general form of multivariate skew-symmetric distribution is given by the following lemma.

Lemma 1.1. Let $f$ be a p.d.f. of a p-dimensional random vector symmetric about $\mathbf{0}$, and $G$ an absolutely continuous distribution function such that $G^{\prime}$ is symmetric about 0 . Then

$$
f_{Z}(z ; \boldsymbol{\alpha})=2 f(\boldsymbol{z}) G\left(\boldsymbol{\alpha}^{\prime} \boldsymbol{z}\right), \quad \boldsymbol{z} \in \mathbb{R}^{p},
$$

is a p.d.f. of a random p-vector $\boldsymbol{Z}$ for any $\boldsymbol{\alpha} \in \mathbb{R}^{p}$.

Note that in Lemma 1.1, for $f_{\boldsymbol{Z}}(\boldsymbol{z})$ being a p.d.f., the condition for $G$ may not be needed. For some $k>0,0 \leqslant G(x) \leqslant k, G^{\prime}(x)$ exists, and $G(x)+G(-x)=1, \forall x \in \mathbb{R}^{1}$, is enough. For example, if $G(x)=\frac{1}{2}, \forall x \in \mathbb{R}^{1}$, then $f_{Z}(z ; \boldsymbol{\alpha})=f(\boldsymbol{z}), \boldsymbol{z} \in \mathbb{R}^{p}$, is a p.d.f. Also the multivariate skew normal distribution is one special case of the general form, which is obtained by taking $f \equiv \phi_{p}$ and $G \equiv \Phi$ in (2).

In this paper, we consider a class of multivariate skew-symmetric distributions generated by the normal kernel. We only take $f \equiv \phi_{p}$ in (2), and let $G$ be defined as in Lemma 1.1. Namely, we say $\boldsymbol{Z}$ has a multivariate skew normal-symmetric distribution, if the p.d.f. of $\boldsymbol{Z}$ is given by

$$
f_{Z}(z)=2 \phi_{p}(z ; \boldsymbol{\Omega}) G\left(\alpha^{\prime} z\right), \quad z \in \mathbb{R}^{p},
$$

for some $\alpha \in \mathbb{R}^{p}$.

Gupta and Huang (2002) have studied quadratic forms of multivariate skew normal-normal model. That is they consider the case $G \equiv \Phi$ in (3). In this paper, we will obtain some parallel results for the class of multivariate skew normal-symmetric distributions. In Section 2, we discuss the moment generating function (m.g.f.) of the quadratic form, $\boldsymbol{Q}=(\boldsymbol{Z}-\boldsymbol{a})^{\prime} A(\boldsymbol{Z}-\boldsymbol{a})$, where $\boldsymbol{a} \in \mathbb{R}^{p}, \boldsymbol{A}$ is a $p \times p$ symmetric matrix, and $\boldsymbol{Z}$ has a multivariate skew normal-symmetric distribution with p.d.f. given in (3). In Section 3, the independence of a linear compound and a quadratic form, and two quadratic forms are studied. Then in the following sections, we discuss some skewed models generated by a normal kernel: the multivariate skew normal-normal model (Section 4), the multivariate skew normal-Laplace model (Section 5), the multivariate skew normal-logistic model (Section 6) and the multivariate skew normal-uniform model (Section 7). The reason that we do not consider the normal- $t$ and normal-Cauchy models, which are also studied in Nadarajah and Kotz (2003), is that the closed form of the m.g.f. of $\boldsymbol{Q}$ cannot be obtained in either case.

Note that when $\boldsymbol{Z}$ has the p.d.f. (3), the m.g.f. of $\boldsymbol{Z}$ is

$$
M_{Z}(\boldsymbol{t})=E\left(e^{\boldsymbol{t}^{\prime} \boldsymbol{Z}}\right)=2 \exp \left\{\frac{1}{2} \boldsymbol{t}^{\prime} \boldsymbol{\Omega} \boldsymbol{t}\right\} E_{\boldsymbol{U}}\left[G\left(\boldsymbol{\alpha}^{\prime} \boldsymbol{U}+\boldsymbol{\alpha}^{\prime} \boldsymbol{\Omega} \boldsymbol{t}\right)\right], \quad \boldsymbol{t} \in \mathbb{R}^{p},
$$

and the m.g.f. of the linear form $\boldsymbol{h}^{\prime} \boldsymbol{Z}, \boldsymbol{h} \in \mathbb{R}^{p}$, is

$$
M_{\boldsymbol{h}^{\prime} \boldsymbol{Z}}(t)=E\left[e^{t \boldsymbol{h}^{\prime} \boldsymbol{Z}}\right]=M_{\boldsymbol{Z}}[t \boldsymbol{h}]=2 \exp \left\{\frac{1}{2} t^{2} \boldsymbol{h}^{\prime} \boldsymbol{\Omega} \boldsymbol{h}\right\} E_{\boldsymbol{U}}\left[G\left(\boldsymbol{\alpha}^{\prime} \boldsymbol{U}+t \boldsymbol{\alpha}^{\prime} \boldsymbol{\Omega} \boldsymbol{h}\right)\right], \quad t \in \mathbb{R}^{1},
$$

where $\boldsymbol{U} \sim N_{p}(\mathbf{0}, \boldsymbol{\Omega})$.

Throughout the rest of this paper, let $\boldsymbol{a} \in \mathbb{R}^{p}, \boldsymbol{A}^{\prime}=\boldsymbol{A}$, a $p \times p$ matrix. 


\section{Moment generating functions of certain quadratic forms}

In this section, let $Z$ be a multivariate skew normal-symmetric distribution with p.d.f. given in (3). First we derive the m.g.f. of the quadratic form $\boldsymbol{Q}=(\boldsymbol{Z}-\boldsymbol{a})^{\prime} \boldsymbol{A}(\boldsymbol{Z}-\boldsymbol{a})$.

Theorem 2.1. The m.g.f. of $\boldsymbol{Q}$ is given by

$$
\begin{aligned}
M_{\boldsymbol{Q}}(t)= & \frac{2 \exp \left\{\boldsymbol{a}^{\prime}\left[t \boldsymbol{A}+2 t^{2} \boldsymbol{A}\left(\boldsymbol{\Omega}^{-1}-2 t \boldsymbol{A}\right)^{-1} \boldsymbol{A}\right] \boldsymbol{a}\right\}}{|\boldsymbol{I}-2 t \boldsymbol{A} \boldsymbol{\Omega}|^{1 / 2}} \\
& \times E_{\boldsymbol{U}_{1}}\left[G\left(-2 t \boldsymbol{\alpha}^{\prime}\left(\boldsymbol{\Omega}^{-1}-2 t \boldsymbol{A}\right)^{-1} \boldsymbol{A} \boldsymbol{a}+\boldsymbol{\alpha}^{\prime}\left(\boldsymbol{\Omega}^{-1}-2 t \boldsymbol{A}\right)^{-1 / 2} \boldsymbol{U}_{1}\right)\right], \quad t \in \mathbb{R}^{1},
\end{aligned}
$$

where $\boldsymbol{U}_{1} \sim N_{p}(\mathbf{0}, \boldsymbol{I})$.

Proof. For $t \in \mathbb{R}^{1}$, the m.g.f. of $\boldsymbol{Q}$ is

$$
\begin{aligned}
M_{\boldsymbol{Q}}(t)= & E\left(e^{t \boldsymbol{Q}}\right) \\
= & 2 \int_{\mathbb{R}^{p}} \exp \left\{t(\boldsymbol{z}-\boldsymbol{a})^{\prime} \boldsymbol{A}(\boldsymbol{z}-\boldsymbol{a})\right\} \phi_{p}(\boldsymbol{z} ; \boldsymbol{\Omega}) G\left(\boldsymbol{\alpha}^{\prime} \boldsymbol{z}\right) \mathrm{d} \boldsymbol{z} \\
= & 2 \int_{\mathbb{R}^{p}} \exp \left\{-\frac{1}{2}\left(\boldsymbol{z}^{\prime} \boldsymbol{\Omega}^{-1} \boldsymbol{z}-2 t(\boldsymbol{z}-\boldsymbol{a})^{\prime} \boldsymbol{A}(\boldsymbol{z}-\boldsymbol{a})\right)\right\} G\left(\boldsymbol{\alpha}^{\prime} \boldsymbol{z}\right) \mathrm{d} \boldsymbol{z} \\
= & \frac{2 \exp \left\{\boldsymbol{a}^{\prime}\left[t \boldsymbol{A}+2 t^{2} \boldsymbol{A}\left(\boldsymbol{\Omega}^{-1}-2 t \boldsymbol{A}\right)^{-1} \boldsymbol{A}\right] \boldsymbol{a}\right\}}{|\boldsymbol{I}-2 t \boldsymbol{A} \boldsymbol{\Omega}|^{1 / 2}} \\
& \times E_{\boldsymbol{U}_{1}}\left[G\left(-2 t \boldsymbol{\alpha}^{\prime}\left(\boldsymbol{\Omega}^{-1}-2 t \boldsymbol{A}\right)^{-1} \boldsymbol{A} \boldsymbol{a}+\boldsymbol{\alpha}^{\prime}\left(\boldsymbol{\Omega}^{-1}-2 t \boldsymbol{A}\right)^{-1 / 2} \boldsymbol{U}_{1}\right)\right], \quad t \in \mathbb{R}^{1} .
\end{aligned}
$$

We also need the following two preliminary lemmas.

Lemma 2.2. Let $X$ be a normally distributed random variable with mean zero. Let $F(y)$ be a continuous function which satisfies $F(y)+F(-y)=1, y \in \mathbb{R}^{1}$. Then

$$
E[F(X)]=\frac{1}{2} \text {. }
$$

Lemma 2.3. If $\boldsymbol{X} \sim N_{n}(\boldsymbol{\mu}, \boldsymbol{\Sigma})$, where $\boldsymbol{C}$ is an $n \times m$ constant matrix, $m \leqslant n$, and $\boldsymbol{V}$ is an $m \times 1$ constant vector, then $\boldsymbol{Y}=\boldsymbol{C}^{\prime} \boldsymbol{X}+\boldsymbol{V} \sim N_{m}\left(\boldsymbol{C}^{\prime} \boldsymbol{\mu}+\boldsymbol{V}, \boldsymbol{C}^{\prime} \boldsymbol{\Sigma} \boldsymbol{C}\right)$. Specially, if $m=1$, i.e. $\boldsymbol{C}=\boldsymbol{c}$ is an $n \times 1$ column vector, and let $\boldsymbol{V}=\mathbf{0}$, then $\boldsymbol{Y}=\boldsymbol{c}^{\prime} \boldsymbol{X} \sim \mathrm{N}\left(\boldsymbol{c}^{\prime} \boldsymbol{\mu}, \boldsymbol{c}^{\prime} \boldsymbol{\Sigma} \boldsymbol{c}\right)$.

By using the above results, the following corollary gives the distribution of four special quadratic forms.

Corollary 2.4. Let $M_{\boldsymbol{Q}_{i}}(t)=E\left(e^{t \boldsymbol{Q}_{i}}\right), t \in \mathbb{R}^{1}, i=1,2,3,4$.

(i) Let $\boldsymbol{Q}_{1}=\boldsymbol{Z}^{\prime} \boldsymbol{A} \boldsymbol{Z}$, where $\boldsymbol{A} \boldsymbol{\Omega}=\operatorname{diag}\left(\delta_{1}, \ldots, \delta_{p}\right)$. Then $\boldsymbol{Z}^{\prime} \boldsymbol{A} \boldsymbol{Z} \backsim \sum_{j=1}^{p} \delta_{j} X_{j}$, where $X_{j} \backsim \chi_{1}^{2}, j=1, \ldots, p$, are independent and identically distributed (i.i.d.).

(ii) Let $\boldsymbol{Q}_{2}=\boldsymbol{Z}^{\prime} \mathbf{\Omega}^{-1} \boldsymbol{Z}$. Then $\boldsymbol{Z}^{\prime} \boldsymbol{\Omega}^{-1} \boldsymbol{Z} \backsim \chi_{p}^{2}$.

(iii) Let $\boldsymbol{Q}_{3}=(\boldsymbol{Z}-\boldsymbol{a})^{\prime} \boldsymbol{\Omega}^{-1}(\boldsymbol{Z}-\boldsymbol{a})$. Then

$$
M_{\boldsymbol{Q}_{3}}(t)=\frac{2 \exp \left\{(t /(1-2 t)) \boldsymbol{a}^{\prime} \boldsymbol{\Omega}^{-1} \boldsymbol{a}\right\}}{(1-2 t)^{p / 2}} \times E_{\boldsymbol{U}_{1}}\left[G\left(\frac{-2 t}{1-2 t} \boldsymbol{\alpha}^{\prime} \boldsymbol{a}+\frac{1}{(1-2 t)^{1 / 2}} \boldsymbol{\alpha}^{\prime} \mathbf{\Omega}^{1 / 2} \boldsymbol{U}_{1}\right)\right], \quad t \in \mathbb{R}^{1}
$$

(iv) Let $\boldsymbol{Q}_{4}=\boldsymbol{Z}^{\prime} \boldsymbol{A} \boldsymbol{Z}$. Then

$$
M_{\boldsymbol{Q}_{4}}(t)=|\boldsymbol{I}-2 t \boldsymbol{A} \boldsymbol{\Omega}|^{-1 / 2}, \quad \boldsymbol{\Omega}^{-1}-2 t \boldsymbol{A}>0, \quad t \in \mathbb{R}^{1} .
$$


Proof. (i) Substituting $\boldsymbol{a}=\mathbf{0}$, and $\boldsymbol{A} \boldsymbol{\Omega}=\operatorname{diag}\left(\delta_{1}, \ldots, \delta_{p}\right)$ in (6), we obtain the m.g.f. of $\boldsymbol{Q}_{1}$ :

$$
\begin{aligned}
M_{\boldsymbol{Q}_{1}}(t) & =\frac{2}{|\boldsymbol{I}-2 t \boldsymbol{A} \boldsymbol{\Omega}|^{1 / 2}} \times E_{\boldsymbol{U}_{1}}\left[G\left(\boldsymbol{\alpha}^{\prime}\left(\boldsymbol{\Omega}^{-1}-2 t \boldsymbol{A}\right)^{-1 / 2} \boldsymbol{U}_{1}\right)\right] \\
& =\left[\prod_{j=1}^{p}\left(1-2 t \delta_{j}\right)\right]^{-1 / 2}=\prod_{j=1}^{p}\left(1-2 t \delta_{j}\right)^{-1 / 2}, \quad t \in \mathbb{R}^{1},
\end{aligned}
$$

where $E_{\boldsymbol{U}_{1}}\left[G\left(\boldsymbol{\alpha}^{\prime}\left(\boldsymbol{\Omega}^{-1}-2 t \boldsymbol{A}\right)^{-1 / 2} \boldsymbol{U}_{1}\right)\right]=\frac{1}{2}$ is obtained by using Lemma 2.2.

(ii) By case (i), substituting $\boldsymbol{A}=\boldsymbol{\Omega}^{-1}$ such that $\boldsymbol{A} \boldsymbol{\Omega}=\boldsymbol{\Omega}^{-1} \boldsymbol{\Omega}=\boldsymbol{I}$, i.e. $\delta_{j}=1, j=1, \ldots, p$, we obtain the m.g.f. of $\boldsymbol{Q}_{2}$ :

$$
M_{Q_{2}}(t)=\prod_{j=1}^{p}\left(1-2 t \delta_{j}\right)^{-1 / 2}=(1-2 t)^{-p / 2}, \quad t \in \mathbb{R}^{1} .
$$

(iii) Substituting $\boldsymbol{A}=\boldsymbol{\Omega}^{-1}$ in (6), we obtain the m.g.f. of $\boldsymbol{Q}_{3}$ :

$$
\begin{aligned}
M_{\boldsymbol{Q}_{3}}(t)= & \frac{2 \exp \left\{\boldsymbol{a}^{\prime}\left[t \boldsymbol{\Omega}^{-1}+2 t^{2} \boldsymbol{\Omega}^{-1}\left(\boldsymbol{\Omega}^{-1}-2 t \boldsymbol{\Omega}^{-1}\right)^{-1} \boldsymbol{\Omega}^{-1}\right] \boldsymbol{a}\right\}}{\left|\boldsymbol{I}-2 t \boldsymbol{\Omega}^{-1} \boldsymbol{\Omega}\right|^{1 / 2}} \\
& \times E_{\boldsymbol{U}_{1}}\left[G\left(-2 t \boldsymbol{\alpha}^{\prime}\left(\boldsymbol{\Omega}^{-1}-2 t \boldsymbol{\Omega}^{-1}\right)^{-1} \boldsymbol{\Omega}^{-1} \boldsymbol{a}+\boldsymbol{\alpha}^{\prime}\left(\boldsymbol{\Omega}^{-1}-2 t \boldsymbol{\Omega}^{-1}\right)^{-1 / 2} \boldsymbol{U}_{1}\right)\right] \\
= & \frac{2 \exp \left\{(t /(1-2 t)) \boldsymbol{a}^{\prime} \boldsymbol{\Omega}^{-1} \boldsymbol{a}\right\}}{(1-2 t)^{p / 2}} \times E_{\boldsymbol{U}_{1}}\left[G\left(\frac{-2 t}{1-2 t} \boldsymbol{\alpha}^{\prime} \boldsymbol{a}+\frac{1}{(1-2 t)^{1 / 2}} \boldsymbol{\alpha}^{\prime} \boldsymbol{\Omega}^{1 / 2} \boldsymbol{U}_{1}\right)\right], \quad t \in \mathbb{R}^{1} .
\end{aligned}
$$

(iv) Substituting $\boldsymbol{a}=\mathbf{0}$ in (6), we obtain the m.g.f. of $\boldsymbol{Q}_{4}$ :

$$
M_{\boldsymbol{Q}_{4}}(t)=|\boldsymbol{I}-2 t \boldsymbol{A} \boldsymbol{\Omega}|^{-1 / 2}, \quad \mathbf{\Omega}^{-1}-2 t \boldsymbol{A}>0, \quad t \in \mathbb{R}^{1} .
$$

Note that $M_{Q_{1}}(t), M_{Q_{2}}(t)$ and $M_{Q_{4}}(t)$ are the same as those in Gupta and Huang (2002), respectively, where multivariate skew normal-normal model is considered. In other words, the distributions of the three quadratic forms $\boldsymbol{Q}_{1}, \boldsymbol{Q}_{2}$ and $\boldsymbol{Q}_{4}$, are independent of $G$. In particular, the distribution of $\boldsymbol{Q}_{4}=\boldsymbol{Z}^{\prime} \boldsymbol{A} \boldsymbol{Z}$ is the same as that of the corresponding quadratic form where $Z \sim N_{p}(\mathbf{0}, \boldsymbol{\Omega})$. Also from (iv), it is easy to obtain the following more general result than (ii) (see Proposition 7 of Azzalini and Capitanio (1999)).

Corollary 2.5. Let $\boldsymbol{B}$ be a symmetric positive semidefinite $p \times p$ matrix of rank $k$ such that $\boldsymbol{B} \boldsymbol{\Omega} \boldsymbol{B}=\boldsymbol{B}$. Then $\boldsymbol{Z}^{\prime} \boldsymbol{B} \boldsymbol{Z} \sim \chi_{k}^{2}$.

\section{Independence of linear forms and quadratic forms}

Theorem 3.1. For $\boldsymbol{h} \in \mathbb{R}^{p}$, if $\boldsymbol{A} \boldsymbol{\Omega} \boldsymbol{h}=0$ and $\boldsymbol{A} \boldsymbol{\Omega} \boldsymbol{\alpha}=0$, the linear form $\boldsymbol{h}^{\prime} \boldsymbol{Z}$ and the quadratic form $\boldsymbol{Z}^{\prime} \boldsymbol{A} \boldsymbol{Z}$ are independent.

Proof. The joint m.g.f. of $\boldsymbol{h}^{\prime} \boldsymbol{Z}$ and $\boldsymbol{Z}^{\prime} \boldsymbol{A} \boldsymbol{Z}$ is

$$
\begin{aligned}
M_{1}\left(t_{1}, t_{2}\right)= & 2 \int_{\mathbb{R}^{p}} \frac{\exp \left\{-\frac{1}{2}\left[\boldsymbol{z}^{\prime} \boldsymbol{\Omega}^{-1} \boldsymbol{z}-2 t_{1} \boldsymbol{h}^{\prime} \boldsymbol{z}-2 t_{2} \boldsymbol{z}^{\prime} \boldsymbol{A} \boldsymbol{z}\right]\right\}}{(2 \pi)^{p / 2}|\boldsymbol{\Omega}|^{1 / 2}} G\left(\boldsymbol{\alpha}^{\prime} \boldsymbol{z}\right) \mathrm{d} \boldsymbol{z} \\
= & \frac{2 \exp \left\{\frac{1}{2} t_{1}^{2} \boldsymbol{h}^{\prime}\left(\boldsymbol{\Omega}^{-1}-2 t_{2} \boldsymbol{A}\right)^{-1} \boldsymbol{h}\right\}}{\left|\boldsymbol{I}-2 t_{2} \boldsymbol{A} \boldsymbol{\Omega}\right|^{1 / 2}} \\
& \times E_{\boldsymbol{U}_{1}}\left[G\left(t_{1} \boldsymbol{\alpha}^{\prime}\left(\boldsymbol{\Omega}^{-1}-2 t_{2} \boldsymbol{A}\right)^{-1} \boldsymbol{h}+\boldsymbol{\alpha}^{\prime}\left(\boldsymbol{\Omega}^{-1}-2 t_{2} \boldsymbol{A}\right)^{-1 / 2} \boldsymbol{U}_{1}\right)\right], \quad t_{1}, t_{2} \in \mathbb{R}^{1} .
\end{aligned}
$$

By Lemma 2.3, we have

$$
\begin{aligned}
& t_{1} \boldsymbol{\alpha}^{\prime}\left(\boldsymbol{\Omega}^{-1}-2 t_{2} \boldsymbol{A}\right)^{-1} \boldsymbol{h}+\boldsymbol{\alpha}^{\prime}\left(\boldsymbol{\Omega}^{-1}-2 t_{2} \boldsymbol{A}\right)^{-1 / 2} \boldsymbol{U}_{1} \\
& \quad \sim \mathrm{N}\left(t_{1} \boldsymbol{\alpha}^{\prime}\left(\boldsymbol{\Omega}^{-1}-2 t_{2} \boldsymbol{A}\right)^{-1} \boldsymbol{h}, \boldsymbol{\alpha}^{\prime}\left(\boldsymbol{\Omega}^{-1}-2 t_{2} \boldsymbol{A}\right)^{-1} \boldsymbol{\alpha}\right) .
\end{aligned}
$$


Now note that

$$
\left(\boldsymbol{\Omega}^{-1}-2 t_{2} \boldsymbol{A}\right)^{-1}=\boldsymbol{\Omega} \sum_{j=0}^{\infty}\left(2 t_{2}\right)^{j}(\boldsymbol{A} \boldsymbol{\Omega})^{j},
$$

for $\left\|2 t_{2} \boldsymbol{A} \boldsymbol{\Omega}\right\|<1$, where $\|\cdot\|$ is a matrix norm. Hence the expansion (9) is always valid in the neighborhood of $t_{2}=0$ (see Horn and Johnson, 1996, p. 301). Finally from (7)-(9), if $\boldsymbol{A} \boldsymbol{\Omega} \boldsymbol{h}=0$, and $\boldsymbol{A} \boldsymbol{\Omega} \boldsymbol{\alpha}=0$, it yields

$$
M_{1}\left(t_{1}, t_{2}\right)=\frac{2 \exp \left\{\frac{1}{2} t_{1}^{2} \boldsymbol{h}^{\prime} \mathbf{\Omega} \boldsymbol{h}\right\}}{\left|\boldsymbol{I}-2 t_{2} \boldsymbol{A} \boldsymbol{\Omega}\right|^{1 / 2}} \times E_{\boldsymbol{U}_{1}}\left[G\left(t_{1} \boldsymbol{\alpha}^{\prime} \boldsymbol{\Omega} \boldsymbol{h}+\boldsymbol{\alpha}^{\prime} \mathbf{\Omega}^{-1 / 2} \boldsymbol{U}_{1}\right)\right], \quad t_{1}, t_{2} \in \mathbb{R}^{1},
$$

which in turn implies $\boldsymbol{h}^{\prime} \boldsymbol{Z}$ and $\boldsymbol{Z}^{\prime} \boldsymbol{A} \boldsymbol{Z}$ are independent.

For the normal-normal model, it is shown in Gupta and Huang (2002), $\boldsymbol{h}^{\prime} \boldsymbol{Z}$ and $\boldsymbol{Z}^{\prime} \boldsymbol{A} \boldsymbol{Z}$ are independent if and only if $\boldsymbol{A} \boldsymbol{\Omega} \boldsymbol{h}=0$ and $\boldsymbol{A} \boldsymbol{\Omega} \boldsymbol{\alpha}=0$. Yet for the general model, we only can show the if part. The following theorem nevertheless is in if and only if form.

Theorem 3.2. Let $\boldsymbol{B}_{1}$ and $\boldsymbol{B}_{2}$ be $p \times p$ symmetric matrices. The quadratic forms $\boldsymbol{Z}^{\prime} \boldsymbol{B}_{1} \boldsymbol{Z}$ and $\boldsymbol{Z}^{\prime} \boldsymbol{B}_{2} \boldsymbol{Z}$ are independent if and only if $\boldsymbol{B}_{1} \boldsymbol{\Omega} \boldsymbol{B}_{2}=0$.

Proof. The joint m.g.f. of $\boldsymbol{Z}^{\prime} \boldsymbol{B}_{1} \boldsymbol{Z}$ and $\boldsymbol{Z}^{\prime} \boldsymbol{B}_{2} \boldsymbol{Z}$ is

$$
\begin{aligned}
M_{2}\left(t_{1}, t_{2}\right) & =2 \int_{\mathbb{R}^{p}} \frac{\exp \left\{-\frac{1}{2}\left[\boldsymbol{z}^{\prime} \mathbf{\Omega}^{-1} \boldsymbol{z}-2 t_{1} \boldsymbol{z}^{\prime} \boldsymbol{B}_{1} \boldsymbol{z}-2 t_{2} \boldsymbol{z}^{\prime} \boldsymbol{B}_{2} \boldsymbol{z}\right]\right\}}{(2 \pi)^{p / 2}|\boldsymbol{\Omega}|^{1 / 2}} G\left(\boldsymbol{\alpha}^{\prime} \boldsymbol{z}\right) \mathrm{d} \boldsymbol{z} \\
& =\frac{2}{|\boldsymbol{\Omega}|^{1 / 2}\left|\boldsymbol{\Omega}^{-1}-2 t_{1} \boldsymbol{B}_{1}-2 t_{2} \boldsymbol{B}_{2}\right|^{1 / 2}} E_{\boldsymbol{U}_{1}}\left(G\left(\boldsymbol{\alpha}^{\prime}\left(\boldsymbol{\Omega}^{-1}-2 t_{1} \boldsymbol{B}_{1}-2 t_{2} \boldsymbol{B}_{2}\right)^{-1 / 2} \boldsymbol{U}_{1}\right)\right) \\
& =\left|\boldsymbol{I}-2\left(t_{1} \boldsymbol{B}_{1}+t_{2} \boldsymbol{B}_{2}\right) \boldsymbol{\Omega}\right|^{-1 / 2}, \quad t_{1}, t_{2} \in \mathbb{R}^{1} .
\end{aligned}
$$

Again the last step is obtained by using Lemma 2.2. Now from (11) for independence we get the condition $\boldsymbol{B}_{1} \boldsymbol{\Omega} \boldsymbol{B}_{2}=0$.

Corollary 3.3. Let $\boldsymbol{B}_{1}, \ldots, \boldsymbol{B}_{n}$ be $p \times p$ symmetric matrices. The quadratic forms $\boldsymbol{Z}^{\prime} \boldsymbol{B}_{i} \boldsymbol{Z}, i=1, \ldots, n$, are mutually independent if and only if $\boldsymbol{B}_{i} \mathbf{\Omega} \boldsymbol{B}_{j}=0, i \neq j$.

By (11), $M_{2}\left(t_{1}, t_{2}\right)$ is independent of $\boldsymbol{\alpha}$. Hence as in Proposition 2 of Loperfido (2001), we have the following consequence.

Corollary 3.4. Let $\boldsymbol{B}_{1}, \ldots, \boldsymbol{B}_{n}$ be $p \times p$ symmetric matrices. The joint distribution of the quadratic forms $\left(\boldsymbol{Z}^{\prime} \boldsymbol{B}_{1} \boldsymbol{Z}, \ldots, \boldsymbol{Z}^{\prime} \boldsymbol{B}_{n} \boldsymbol{Z}\right), i=1, \ldots, n$, does not depend on $\boldsymbol{\alpha}$.

\section{Multivariate skew normal-normal model}

Let $G=\Phi$, then we obtain the multivariate skew normal-normal distribution for $\boldsymbol{Z}$. As mentioned it before, this distribution has been studied by Azzalini and Dalla Valle (1996), its applications are given in Azzalini and Capitanio (1999), and its quadratic form has been studied by Gupta and Huang (2002).

\subsection{M.G.F. of $(Z-a)^{\prime} A(Z-a)$}

In this section we derive the m.g.f. of the quadratic form $\boldsymbol{Q}=(\boldsymbol{Z}-\boldsymbol{a})^{\prime} \boldsymbol{A}(\boldsymbol{Z}-\boldsymbol{a})$. For this we need the following lemma (see Zacks, 1981, pp. 53-59).

Lemma 4.1. Let $\boldsymbol{U} \sim N_{p}(\mathbf{0}, \boldsymbol{\Omega})$. Then, for any scalar $u$ and $\boldsymbol{v} \in \mathbb{R}^{p}$, we have

$$
E\left[\Phi\left(u+\boldsymbol{v}^{\prime} \boldsymbol{U}\right)\right]=\Phi\left\{\frac{u}{\left(1+\boldsymbol{v}^{\prime} \boldsymbol{\Omega} \boldsymbol{v}\right)^{1 / 2}}\right\} .
$$

Using the above lemma, we obtain the following theorem (Theorem 2 of Gupta and Huang, 2002). 
Theorem 4.2. The m.g.f. of $\boldsymbol{Q}$ is given by

$$
M_{\boldsymbol{Q}}(t)=\frac{2 \exp \left\{\boldsymbol{a}^{\prime}\left[t \boldsymbol{A}+2 t^{2} \boldsymbol{A}\left(\boldsymbol{\Omega}^{-1}-2 t \boldsymbol{A}\right)^{-1} \boldsymbol{A}\right] \boldsymbol{a}\right\}}{|\boldsymbol{I}-2 t \boldsymbol{A} \boldsymbol{\Omega}|^{1 / 2}} \times \Phi\left[-\frac{2 t \boldsymbol{\alpha}^{\prime}\left(\boldsymbol{\Omega}^{-1}-2 t \boldsymbol{A}\right)^{-1} \boldsymbol{A} \boldsymbol{a}}{\left(1+\boldsymbol{\alpha}^{\prime}\left(\boldsymbol{\Omega}^{-1}-2 t \boldsymbol{A}\right)^{-1} \boldsymbol{\alpha}\right)^{1 / 2}}\right], \quad t \in \mathbb{R}^{1} .
$$

\subsubsection{Special case}

Case (iii). The m.g.f. of $\boldsymbol{Q}_{3}$ is

$$
M_{\boldsymbol{Q}_{3}}(t)=\frac{2 \exp \left\{(t /(1-2 t)) \boldsymbol{a}^{\prime} \mathbf{\Omega}^{-1} \boldsymbol{a}\right\}}{(1-2 t)^{p / 2}} \times \Phi\left[\frac{-2 t}{1-2 t} \frac{\boldsymbol{\alpha}^{\prime} \boldsymbol{a}}{\left(1+\boldsymbol{\alpha}^{\prime} \mathbf{\Omega} \boldsymbol{\alpha} /(1-2 t)\right)^{1 / 2}}\right], \quad t \in \mathbb{R}^{1} .
$$

\section{Multivariate skew normal-Laplace model}

Let $G=G_{2}$ be the c.d.f. of a Laplace distribution, namely

$$
G_{2}(x)= \begin{cases}1-\frac{1}{2} \exp (-x), & x \geqslant 0 \\ \frac{1}{2} \exp (x), & x<0\end{cases}
$$

we obtain the multivariate skew normal-Laplace distribution for $\boldsymbol{Z}$.

\subsection{M.G.F. of $(Z-a)^{\prime} A(Z-a)$}

In the following we derive the m.g.f. of the quadratic form $\boldsymbol{Q}=(\boldsymbol{Z}-\boldsymbol{a})^{\prime} \boldsymbol{A}(\boldsymbol{Z}-\boldsymbol{a})$. For this we need the following lemma which can be obtained by routine computation.

Lemma 5.1. Let $\boldsymbol{U} \sim N_{p}(\mathbf{0}, \boldsymbol{\Omega})$. Then, for any scalar $u$ and $\boldsymbol{v} \in \mathbb{R}^{p}$, we have

$$
\begin{aligned}
E\left[G_{2}\left(u+\boldsymbol{v}^{\prime} \boldsymbol{U}\right)\right]= & \frac{1}{2} \exp \left\{u+\frac{1}{2} \boldsymbol{v}^{\prime} \boldsymbol{\Omega} \boldsymbol{v}\right\} \Phi\left(\frac{-\left(u+\boldsymbol{v}^{\prime} \boldsymbol{\Omega} \boldsymbol{v}\right)}{\left(\boldsymbol{v}^{\prime} \boldsymbol{\Omega} \boldsymbol{v}\right)^{1 / 2}}\right)+\Phi\left(\frac{u}{\left(\boldsymbol{v}^{\prime} \boldsymbol{\Omega} \boldsymbol{v}\right)^{1 / 2}}\right) \\
& -\frac{1}{2} \exp \left\{-u+\frac{1}{2} \boldsymbol{v}^{\prime} \boldsymbol{\Omega} \boldsymbol{v}\right\} \Phi\left(\frac{u-\boldsymbol{v}^{\prime} \boldsymbol{\Omega} \boldsymbol{v}}{\left(\boldsymbol{v}^{\prime} \boldsymbol{\Omega} \boldsymbol{v}\right)^{1 / 2}}\right) .
\end{aligned}
$$

Substituting (14) in (6) yields the following theorem.

Theorem 5.2. The m.g.f. of $\boldsymbol{Q}$ is given by

$$
\begin{aligned}
& M_{\boldsymbol{Q}}(t)=\frac{2 \exp \left\{\boldsymbol{a}^{\prime}\left[t \boldsymbol{A}+2 t^{2} \boldsymbol{A}\left(\boldsymbol{\Omega}^{-1}-2 t \boldsymbol{A}\right)^{-1} \boldsymbol{A}\right] \boldsymbol{a}\right\}}{|\boldsymbol{I}-2 t \boldsymbol{A} \boldsymbol{\Omega}|^{1 / 2}} \\
& \times\left[\frac{1}{2} \exp \left\{-2 t \boldsymbol{\alpha}^{\prime}\left(\boldsymbol{\Omega}^{-1}-2 t \boldsymbol{A}\right)^{-1} \boldsymbol{A} \boldsymbol{a}+\frac{1}{2} \boldsymbol{\alpha}^{\prime}\left(\boldsymbol{\Omega}^{-1}-2 t \boldsymbol{A}\right)^{-1} \boldsymbol{\alpha}\right\}\right. \\
& \times \Phi\left(\frac{2 t \boldsymbol{\alpha}^{\prime}\left(\boldsymbol{\Omega}^{-1}-2 t \boldsymbol{A}\right)^{-1} \boldsymbol{A} \boldsymbol{a}-\boldsymbol{\alpha}^{\prime}\left(\boldsymbol{\Omega}^{-1}-2 t \boldsymbol{A}\right)^{-1} \boldsymbol{\alpha}}{\left(\boldsymbol{\alpha}^{\prime}\left(\boldsymbol{\Omega}^{-1}-2 t \boldsymbol{A}\right)^{-1} \boldsymbol{\alpha}\right)^{1 / 2}}\right)+\Phi\left(\frac{-2 t \boldsymbol{\alpha}^{\prime}\left(\boldsymbol{\Omega}^{-1}-2 t \boldsymbol{A}\right)^{-1} \boldsymbol{A} \boldsymbol{a}}{\left(\boldsymbol{\alpha}^{\prime}\left(\boldsymbol{\Omega}^{-1}-2 t \boldsymbol{A}\right)^{-1} \boldsymbol{\alpha}\right)^{1 / 2}}\right) \\
& -\frac{1}{2} \exp \left\{2 t \boldsymbol{\alpha}^{\prime}\left(\boldsymbol{\Omega}^{-1}-2 t \boldsymbol{A}\right)^{-1} \boldsymbol{A} \boldsymbol{a}+\frac{1}{2} \boldsymbol{\alpha}^{\prime}\left(\boldsymbol{\Omega}^{-1}-2 t \boldsymbol{A}\right)^{-1} \boldsymbol{\alpha}\right\} \\
& \left.\times \Phi\left(\frac{-2 t \boldsymbol{\alpha}^{\prime}\left(\boldsymbol{\Omega}^{-1}-2 t \boldsymbol{A}\right)^{-1} \boldsymbol{A} \boldsymbol{a}-\boldsymbol{\alpha}^{\prime}\left(\boldsymbol{\Omega}^{-1}-2 t \boldsymbol{A}\right)^{-1} \boldsymbol{\alpha}}{\left(\boldsymbol{\alpha}^{\prime}\left(\boldsymbol{\Omega}^{-1}-2 t \boldsymbol{A}\right)^{-1} \boldsymbol{\alpha}\right)^{1 / 2}}\right)\right], \quad t \in \mathbb{R}^{1} .
\end{aligned}
$$




\subsubsection{Special case}

Case (iii). The m.g.f. of $\boldsymbol{Q}_{3}$ is

$$
\begin{aligned}
M_{\boldsymbol{Q}_{3}}(t)= & \frac{2 \exp \left\{(t /(1-2 t)) \boldsymbol{a}^{\prime} \mathbf{\Omega}^{-1} \boldsymbol{a}\right\}}{(1-2 t)^{p / 2}} \times\left[\Phi\left(\frac{(-2 t /(1-2 t)) \boldsymbol{\alpha}^{\prime} \boldsymbol{a}}{\left.((1 / 1-2 t)) \boldsymbol{\alpha}^{\prime} \mathbf{\Omega} \boldsymbol{\alpha}\right)^{1 / 2}}\right)\right. \\
& +\frac{1}{2} \exp \left\{-\frac{2 t}{1-2 t} \boldsymbol{\alpha}^{\prime} \boldsymbol{a}+\frac{1}{2(1-2 t)} \boldsymbol{\alpha}^{\prime} \mathbf{\Omega} \boldsymbol{\alpha}\right\} \Phi\left(\frac{(2 t /(1-2 t)) \boldsymbol{\alpha}^{\prime} \boldsymbol{a}-\left((1 /(1-2 t)) \boldsymbol{\alpha}^{\prime} \mathbf{\Omega} \boldsymbol{\alpha}\right.}{\left((1 /(1-2 t))\left(\boldsymbol{\alpha}^{\prime} \mathbf{\Omega} \boldsymbol{\alpha}\right)^{1 / 2}\right.}\right) \\
& \left.-\frac{1}{2} \exp \left\{\frac{2 t}{1-2 t} \boldsymbol{\alpha}^{\prime} \boldsymbol{a}+\frac{1}{2(1-2 t)} \boldsymbol{\alpha}^{\prime} \mathbf{\Omega} \boldsymbol{\alpha}\right\} \Phi\left(\frac{(-2 t /(1-2 t)) \boldsymbol{\alpha}^{\prime} \boldsymbol{a}-(1 /(1-2 t)) \boldsymbol{\alpha}^{\prime} \mathbf{\Omega} \boldsymbol{\alpha}}{\left((1 /(1-2 t)) \boldsymbol{\alpha}^{\prime} \mathbf{\Omega} \boldsymbol{\alpha}\right)^{1 / 2}}\right)\right], \quad t \in \mathbb{R}^{1} .
\end{aligned}
$$

\section{Multivariate skew normal-logistic model}

Let $G=G_{3}$ be the c.d.f. of a logistic distribution, namely

$$
G_{3}(x)=\frac{1}{1+\exp (-x / \beta)}, \quad-\infty<x<\infty,
$$

we obtain the multivariate skew normal-logistic distribution for $\boldsymbol{Z}$. Using the Taylor series expansion for $(1+w)^{-1}$, then

$$
G_{3}(x)= \begin{cases}\sum_{j=0}^{\infty}\left(\begin{array}{c}
-1 \\
j
\end{array}\right) \exp \left(-\frac{j x}{\beta}\right), & x \geqslant 0, \\
\exp \left(\frac{x}{\beta}\right) \sum_{j=0}^{\infty}\left(\begin{array}{c}
-1 \\
j
\end{array}\right) \exp \left(\frac{j x}{\beta}\right), & x<0 .\end{cases}
$$

\subsection{M.G.F. of $(Z-a)^{\prime} A(Z-a)$}

In the following we derive the m.g.f. of the quadratic form $\boldsymbol{Q}=(\boldsymbol{Z}-\boldsymbol{a})^{\prime} \boldsymbol{A}(\boldsymbol{Z}-\boldsymbol{a})$. First we need a lemma given by Nadarajah and Kotz (2003).

Lemma 6.1. Let $\boldsymbol{U} \sim N_{p}(\mathbf{0}, \boldsymbol{\Omega})$. Then, for any scalar $u$ and $\boldsymbol{v} \in \mathbb{R}^{p}$, we have

$$
\begin{aligned}
E\left[G_{3}\left(u+\boldsymbol{v}^{\prime} \boldsymbol{U}\right)\right]= & \sum_{j=0}^{\infty}\left(\begin{array}{c}
-1 \\
j
\end{array}\right)\left[\exp \left\{-\frac{j}{\beta} u+\frac{j^{2}}{2 \beta^{2}} \boldsymbol{v}^{\prime} \boldsymbol{\Omega} \boldsymbol{v}\right\} \Phi\left(\frac{u-(j / \beta) \boldsymbol{v}^{\prime} \boldsymbol{\Omega} \boldsymbol{v}}{\left(\boldsymbol{v}^{\prime} \boldsymbol{\Omega} \boldsymbol{v}\right)^{1 / 2}}\right)\right. \\
& \left.+\exp \left\{\frac{(j+1)}{\beta} u+\frac{(j+1)^{2}}{2 \beta^{2}} \boldsymbol{v}^{\prime} \boldsymbol{\Omega} \boldsymbol{v}\right\} \Phi\left(\frac{-u-((j+1) / \beta) \boldsymbol{v}^{\prime} \boldsymbol{\Omega} \boldsymbol{v}}{\left(\boldsymbol{v}^{\prime} \boldsymbol{\Omega} \boldsymbol{v}\right)^{1 / 2}}\right)\right] .
\end{aligned}
$$

Substituting (16) into (6), we obtain the following theorem.

Theorem 6.2. The m.g.f. of $\boldsymbol{Q}$ is given by

$$
\begin{aligned}
M_{\boldsymbol{Q}}(t)= & \frac{2 \exp \left\{\boldsymbol{a}^{\prime}\left[t \boldsymbol{A}+2 t^{2} \boldsymbol{A}\left(\boldsymbol{\Omega}^{-1}-2 t \boldsymbol{A}\right)^{-1} \boldsymbol{A}\right] \boldsymbol{a}\right\}}{|\boldsymbol{I}-2 t \boldsymbol{A} \boldsymbol{\Omega}|^{1 / 2}} \\
& \times \sum_{j=0}^{\infty}\left(\begin{array}{c}
-1 \\
j
\end{array}\right)\left[\exp \left\{\frac{2 t j}{\beta} \boldsymbol{\alpha}^{\prime}\left(\boldsymbol{\Omega}^{-1}-2 t \boldsymbol{A}\right)^{-1} \boldsymbol{A} \boldsymbol{a}+\frac{j^{2}}{2 \beta^{2}} \boldsymbol{\alpha}^{\prime}\left(\boldsymbol{\Omega}^{-1}-2 t \boldsymbol{A}\right)^{-1} \boldsymbol{\alpha}\right\}\right. \\
& \times \Phi\left(\frac{\left.-2 t \boldsymbol{\alpha}^{\prime}\left(\boldsymbol{\Omega}^{-1}-2 t \boldsymbol{A}\right)^{-1} \boldsymbol{A} \boldsymbol{a}-(j / \beta) \boldsymbol{\alpha}^{\prime}\left(\boldsymbol{\Omega}^{-1}-2 t \boldsymbol{A}\right)^{-1} \boldsymbol{\alpha}\right)}{\left(\boldsymbol{\alpha}^{\prime}\left(\boldsymbol{\Omega}^{-1}-2 t \boldsymbol{A}\right)^{-1} \boldsymbol{\alpha}\right)^{1 / 2}}\right)
\end{aligned}
$$




$$
\begin{aligned}
& +\exp \left\{-\frac{2 t(j+1)}{\beta} \boldsymbol{\alpha}^{\prime}\left(\boldsymbol{\Omega}^{-1}-2 t \boldsymbol{A}\right)^{-1} \boldsymbol{A} \boldsymbol{a}+\frac{(j+1)^{2}}{2 \beta^{2}} \boldsymbol{\alpha}^{\prime}\left(\boldsymbol{\Omega}^{-1}-2 t \boldsymbol{A}\right)^{-1} \boldsymbol{\alpha}\right\} \\
& \left.\times \Phi\left(\frac{2 t \boldsymbol{\alpha}^{\prime}\left(\boldsymbol{\Omega}^{-1}-2 t \boldsymbol{A}\right)^{-1} \boldsymbol{A} \boldsymbol{a}-((j+1) / \beta) \boldsymbol{\alpha}^{\prime}\left(\boldsymbol{\Omega}^{-1}-2 t \boldsymbol{A}\right)^{-1} \boldsymbol{\alpha}}{\left(\boldsymbol{\alpha}^{\prime}\left(\boldsymbol{\Omega}^{-1}-2 t \boldsymbol{A}\right)^{-1} \boldsymbol{\alpha}\right)^{1 / 2}}\right)\right], \quad t \in \mathbb{R}^{1} .
\end{aligned}
$$

\subsubsection{Special case}

Case (iii). The m.g.f. of $\boldsymbol{Q}_{3}$ is

$$
\begin{aligned}
M_{\mathbf{Q}_{3}}(t)= & \frac{2 \exp \left\{(t /(1-2 t)) \boldsymbol{a}^{\prime} \mathbf{\Omega}^{-1} \boldsymbol{a}\right\}}{(1-2 t)^{p / 2}} \times\left[\sum _ { j = 0 } ^ { \infty } ( \begin{array} { c } 
{ - 1 } \\
{ j }
\end{array} ) \left[\exp \left\{\frac{2 t j}{(1-2 t) \beta} \boldsymbol{\alpha}^{\prime} \boldsymbol{a}+\frac{j^{2}}{2(1-2 t) \beta^{2}} \boldsymbol{\alpha}^{\prime} \mathbf{\Omega} \boldsymbol{\alpha}\right\}\right.\right. \\
& \times \Phi\left(\frac{\left.(-2 t /(1-2 t)) \boldsymbol{\alpha}^{\prime} \boldsymbol{a}-(j /(1-2 t) \beta)\right) \boldsymbol{\alpha}^{\prime} \mathbf{\Omega} \boldsymbol{\alpha}}{\left((1 /(1-2 t)) \boldsymbol{\alpha}^{\prime} \mathbf{\Omega} \boldsymbol{\alpha}\right)^{1 / 2}}\right)+\exp \left\{-\frac{2 t(j+1)}{(1-2 t) \beta} \boldsymbol{\alpha}^{\prime} \boldsymbol{a}+\frac{(j+1)^{2}}{2(1-2 t) \beta^{2}} \boldsymbol{\alpha}^{\prime} \mathbf{\Omega} \boldsymbol{\alpha}\right\} \\
& \left.\times \Phi\left(\frac{(2 t /(1-2 t)) \boldsymbol{\alpha}^{\prime} \boldsymbol{a}-((j+1) /((1-2 t) \beta)) \boldsymbol{\alpha}^{\prime} \mathbf{\Omega} \boldsymbol{\alpha}}{\left((1 /(1-2 t)) \boldsymbol{\alpha}^{\prime} \mathbf{\Omega} \boldsymbol{\alpha}\right)^{1 / 2}}\right)\right], \quad t \in \mathbb{R}^{1} .
\end{aligned}
$$

\section{Multivariate skew normal-uniform model}

Let $G=G_{4}$ be the c.d.f. of a uniform distribution, namely

$$
G_{4}(x)= \begin{cases}0, & x<-h, \\ \frac{x+h}{2 h}, & -h \leqslant x<h, \\ 1, & x \geqslant h,\end{cases}
$$

we obtain the multivariate skew normal-uniform distribution for $\boldsymbol{Z}$.

\subsection{M.G.F. of $(Z-a)^{\prime} A(Z-a)$}

In the following we derive the m.g.f. of the quadratic form $\boldsymbol{Q}=(\boldsymbol{Z}-\boldsymbol{a})^{\prime} \boldsymbol{A}(\boldsymbol{Z}-\boldsymbol{a})$. Again first we give a lemma.

Lemma 7.1. Let $\boldsymbol{U} \sim N_{p}(\mathbf{0}, \mathbf{\Omega})$. Then, for any scalar $u$ and $\boldsymbol{v} \in \mathbb{R}^{p}$,

$$
\begin{aligned}
E\left[G_{4}\left(u+\boldsymbol{v}^{\prime} \boldsymbol{U}\right)\right]= & \frac{\left(\boldsymbol{v}^{\prime} \boldsymbol{\Omega v}\right)^{1 / 2}}{2 h \sqrt{2 \pi}} \exp \left\{-\frac{(h+u)^{2}}{2 \boldsymbol{v}^{\prime} \boldsymbol{\Omega} \boldsymbol{v}}\right\}-\frac{\left(\boldsymbol{v}^{\prime} \boldsymbol{\Omega} \boldsymbol{v}\right)^{1 / 2}}{2 h \sqrt{2 \pi}} \exp \left\{-\frac{(h-u)^{2}}{2 \boldsymbol{v}^{\prime} \boldsymbol{\Omega} \boldsymbol{v}}\right\} \\
& +\left(\frac{u}{2 h}-\frac{1}{2}\right)\left[\Phi\left(\frac{h-u}{\left(\boldsymbol{v}^{\prime} \boldsymbol{\Omega} \boldsymbol{v}\right)^{1 / 2}}\right)-1\right]+\left(\frac{u}{2 h}+\frac{1}{2}\right) \Phi\left(\frac{h+u}{\left(\boldsymbol{v}^{\prime} \boldsymbol{\Omega} \boldsymbol{v}\right)^{1 / 2}}\right) .
\end{aligned}
$$

Substituting (18) into (6), we obtain the following theorem.

Theorem 7.2. The m.g.f. of $\boldsymbol{Q}$ is given by

$$
\begin{aligned}
M_{\boldsymbol{Q}}(t)= & \frac{2 \exp \left\{\boldsymbol{a}^{\prime}\left[t \boldsymbol{A}+2 t^{2} \boldsymbol{A}\left(\boldsymbol{\Omega}^{-1}-2 t \boldsymbol{A}\right)^{-1} \boldsymbol{A}\right] \boldsymbol{a}\right\}}{|\boldsymbol{I}-2 t \boldsymbol{A} \boldsymbol{\Omega}|^{1 / 2}} \\
& \times\left[\frac{\left(\boldsymbol{\alpha}^{\prime}\left(\boldsymbol{\Omega}^{-1}-2 t \boldsymbol{A}\right)^{-1} \boldsymbol{\alpha}\right)^{1 / 2}}{2 h \sqrt{2 \pi}} \exp \left\{-\frac{\left(h-2 t \boldsymbol{\alpha}^{\prime}\left(\boldsymbol{\Omega}^{-1}-2 t \boldsymbol{A}\right)^{-1} \boldsymbol{A} \boldsymbol{a}\right)^{2}}{2\left(\boldsymbol{\alpha}^{\prime}\left(\boldsymbol{\Omega}^{-1}-2 t \boldsymbol{A}\right)^{-1} \boldsymbol{\alpha}\right)}\right\}\right.
\end{aligned}
$$




$$
\begin{aligned}
& -\frac{\left(\boldsymbol{\alpha}^{\prime}\left(\boldsymbol{\Omega}^{-1}-2 t \boldsymbol{A}\right)^{-1} \boldsymbol{\alpha}\right)^{1 / 2}}{2 h \sqrt{2 \pi}} \exp \left\{-\frac{\left(h+2 t \boldsymbol{\alpha}^{\prime}\left(\boldsymbol{\Omega}^{-1}-2 t \boldsymbol{A}\right)^{-1} \boldsymbol{A a}\right)^{2}}{2\left(\boldsymbol{\alpha}^{\prime}\left(\boldsymbol{\Omega}^{-1}-2 t \boldsymbol{A}\right)^{-1} \boldsymbol{\alpha}\right)}\right\} \\
& +\left(\frac{-2 t \boldsymbol{\alpha}^{\prime}\left(\boldsymbol{\Omega}^{-1}-2 t \boldsymbol{A}\right)^{-1} \boldsymbol{A a}}{2 h}-\frac{1}{2}\right)\left[\Phi\left(\frac{h+2 t \boldsymbol{\alpha}^{\prime}\left(\boldsymbol{\Omega}^{-1}-2 t \boldsymbol{A}\right)^{-1} \boldsymbol{A a}}{\left(\boldsymbol{\alpha}^{\prime}\left(\boldsymbol{\Omega}^{-1}-2 t \boldsymbol{A}\right)^{-1} \boldsymbol{\alpha}\right)^{1 / 2}}\right)-1\right] \\
& \left.+\left(\frac{-2 t \boldsymbol{\alpha}^{\prime}\left(\boldsymbol{\Omega}^{-1}-2 t \boldsymbol{A}\right)^{-1} \boldsymbol{A a}}{2 h}+\frac{1}{2}\right) \Phi\left(\frac{h-2 t \boldsymbol{\alpha}^{\prime}\left(\boldsymbol{\Omega}^{-1}-2 t \boldsymbol{A}\right)^{-1} \boldsymbol{A a}}{\left(\boldsymbol{\alpha}^{\prime}\left(\boldsymbol{\Omega}^{-1}-2 t \boldsymbol{A}\right)^{-1} \boldsymbol{\alpha}\right)^{1 / 2}}\right)\right], \quad t \in \mathbb{R}^{1} .
\end{aligned}
$$

\subsubsection{Special case}

Case (iii). The m.g.f. of $\boldsymbol{Q}_{3}$ is

$$
\begin{aligned}
M_{\mathbf{Q}_{3}}(t)= & \frac{2 \exp \left\{(t /(1-2 t)) \boldsymbol{a}^{\prime} \mathbf{\Omega}^{-1} \boldsymbol{a}\right\}}{(1-2 t)^{p / 2}} \\
& \times\left\{\frac{\left((1 /(1-2 t)) \boldsymbol{\alpha}^{\prime} \boldsymbol{\Omega} \boldsymbol{\alpha}\right)^{1 / 2}}{2 h \sqrt{2 \pi}}\left(\exp \left\{-\frac{\left(h-(2 t /(1-2 t)) \boldsymbol{\alpha}^{\prime} \boldsymbol{a}\right)^{2}}{(2 /(1-2 t)) \boldsymbol{\alpha}^{\prime} \boldsymbol{\Omega} \boldsymbol{\alpha}}\right\}-\exp \left\{-\frac{\left(h+(2 t /(1-2 t)) \boldsymbol{\alpha}^{\prime} \boldsymbol{a}\right)^{2}}{(2 /(1-2 t)) \boldsymbol{\alpha}^{\prime} \boldsymbol{\Omega} \boldsymbol{\alpha}}\right\}\right)\right. \\
& +\left(\frac{(-2 t /(1-2 t)) \boldsymbol{\alpha}^{\prime} \boldsymbol{a}}{2 h}-\frac{1}{2}\right)\left[\Phi\left(\frac{h+(2 t /(1-2 t)) \boldsymbol{\alpha}^{\prime} \boldsymbol{a}}{\left((1 /(1-2 t)) \boldsymbol{\alpha}^{\prime} \mathbf{\Omega} \boldsymbol{\alpha}\right)^{1 / 2}}\right)-1\right] \\
& \left.+\left(\frac{(-2 t /(1-2 t)) \boldsymbol{\alpha}^{\prime} \boldsymbol{a}}{2 h}+\frac{1}{2}\right) \Phi\left(\frac{h-(2 t /(1-2 t)) \boldsymbol{\alpha}^{\prime} \boldsymbol{a}}{\left((1 /(1-2 t)) \boldsymbol{\alpha}^{\prime} \boldsymbol{\Omega} \boldsymbol{\alpha}\right)^{1 / 2}}\right)\right\}, \quad t \in \mathbb{R}^{1} .
\end{aligned}
$$

\section{References}

Arellano-Valle, R.B., Gómez, H.W., Quintana, F.A., 2004. A new class of skew-normal distributions. Comm. Statist. Theory Methods 33, $1975-1991$.

Arnold, B.C., Beaver, R.J., 2000. The skew-Cauchy distribution. Statist. Probab. Lett. 49, 285-290.

Azzalini, A., 1985. A class of distributions which includes the normal ones. Scand. J. Statist. 12, 171-178.

Azzalini, A., 1986. Further results on a class of distributions which includes the normal ones. Statistica 46, 199-208.

Azzalini, A., Capitanio, A., 1999. Statistical applications of the multivariate skew normal distribution. J. Roy. Statist. Soc. B 61, 579-602.

Azzalini, A., Dalla Valle, A., 1996. The multivariate skew-normal distribution. Biometrika 83, 715-726.

Genton, M.G., He, L., Liu, X., 2001. Moments of skew-normal random vectors and their quadratic forms. Statist. Probab. Lett. 51, $319-325$.

Gupta, A.K., 2003. Multivariate skew t-distribution. Statistics 37, 359-363.

Gupta, A.K., Chang, F.-C., 2003. Multivariate skew-symmetric distributions. Appl. Math. Lett. 16, $643-646$.

Gupta, A.K., Huang, W.-J., 2002. Quadratic forms in skew normal variates. J. Math. Anal. Appl. 273, 558-564.

Gupta, A.K., Kollo, T., 2000. Multivariate skew normal distribution: some properties and density expansions. T.R. No. 00-03, Department of Mathematics and Statistics, Bowling Green State University.

Gupta, A.K., Chang, F.-C., Huang, W.-J., 2002. Some skew-symmetric models. Rand. Oper. Stoch. Equation 10, $133-140$.

Gupta, A.K., González-Farías, G., Domínguez-Molina, J.A., 2004a. A multivariate skew normal distribution. J. Multivariate Anal. 89, $181-190$.

Gupta, A.K., Nguyen, T.T., Sanqui, J.A.T., 2004b. Characterization of the skew-normal distribution. Ann. Inst. Statist. Math. 56, $351-360$.

Horn, R.A., Johnson, C.R., 1996. Matrix Analysis. Cambridge University Press, Cambridge.

Loperfido, N., 2001. Quadratic forms of skew-normal random vectors. Statist. Probab. Lett. 54, 381-387.

Nadarajah, S., Kotz, S., 2003. Skewed distributions generated by the normal kernel. Statist. Probab. Lett. 65, $269-277$.

Zacks, S., 1981. Parametric Statistical Inference. Basic Theory and Modern Approaches. Pergamon Press, Oxford. 